Proceedings

\title{
Can specific pre-workout meals reduce bone resorption in young gymnasts?
}

\author{
Alessandra Amato ${ }^{1}$, Patrizia Proia ${ }^{1}$, Gaetano Felice Caldara ${ }^{2}$, Angelina Alongi ${ }^{3}$, Vincenzo Ferrantelli ${ }^{3}$, Sara Baldas- \\ sano $^{4}$
}

Publisher's Note: MDPI stays neutral with regard to jurisdictional claims in published maps and institutional affiliations.

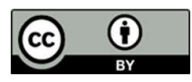

Copyright: $₫ 2020$ by the authors. Submitted for possible open access publication under the terms and conditions of the Creative Commons Attribution (CC BY) license (http://creativecommons.org/licenses/by/4.0/).

\author{
1 Department of Psychological, Pedagogical and Educational Sciences, Sport and Exercise Sciences Re- \\ search Unit \\ Dipartimento di scienze per la promozione della salute e materno-infantile G. D'Alessandro" \\ Istituto Zooprofilattico Sperimentale della Sicilia \\ Department of Biological, Chemical and Pharmaceutical Sciences and Technologies (STEBICEF), Univer- \\ sity of Palermo, Italy
}

\begin{abstract}
The pre-adolescence in woman is a key stage to obtain adequate bone mineral content and to decrease the risk of osteoporosis in adulthood. Exercise and adequate nutrition can influence the bone growth process. The aim of this paper was to verify if a different pre-exercise meal can change exercise-induced bone resorption in female child artistic gymnasts. Twenty-eight preadolescent female were requited for this study. Training was preceded by high-carbohydrate meal (HCM; 300 kcal, $88 \%$ carbohydrates, $9 \%$ protein, 3\% fat) or high protein meal (HPM; $300 \mathrm{kcal}, 55 \%$ carbohydrates, $31 \%$ protein, $13 \%$ fat) ninety minutes before the start of the training session. Bone resorption biomarker, C-terminal telopeptide region of collagen type 1 (CTX), were analyzed from the urine sample collected pre and post meal and post- gymnastics workout. Pre-exercise eating habits were examined by a validated short food frequency question (FFQ) for children. The group with HCM significantly reduces bone resorption post-exercise by decreasing CTX levels more than HPM group. The FFQ shows that about $30 \%$ of the children, of both groups, do not have regularly pre-exercise eating habits. In conclusion, HCM prior to high intensity physical activity seems to reduce acute post-exercise bone resorption in pre-pubertal age gymnasts. The analysis of the pre-exercise eating habits indicate that there is variability in the frequency and timing of meal consumption before exercise.
\end{abstract}

Keywords: Bone resorption; children; meal composition; physical activity; pre-exercise eating habits

\section{Introduction}

Bone mineral density is modified by various factors and in particular by diet, by nutritional status and exercise $(1,2,3)$. Athletes have a higher bone turnover compared to sedentary individuals. Moderate-to-vigorous activity is useful to improve cardiovascular fitness and bone health and to prevent sedentary lifestyle (4). However, prolonged physical exercise at a competitive level can negatively affect bone remodeling and compromise health of the skeleton (5-7). A correct diet that respects the athlete's nutritional needs with respect to physical training regimes is therefore essential to reach puberty in good physical shape (8). The degree of formation and resorption can be assessed by measuring markers of bone resorption in the blood or in the urine (9). These markers are also useful for early monitoring of any changes in the turnover rate of bone in order to prevent bone fragility and osteoporosis. The aim of the study was to study which food, high-carbohydrate meal or high protein meal, provided pre-workout was able to affect exercise-induced bone resorption in female child artistic gymnasts. We also collected information on the eating habits of the young gymnasts. 


\section{Methods}

The study population consisted of twenty-eight pre-pubertal girls. The gymnasts were divided into two groups with half assigned to the protein group and half assigned to the carbohydrate group. A schematic description of the protocol is reported in the figure 1. The participants were interviewed using questionnaires to report food frequency in children. Human Cross-linked C -terminal telopeptide of type I collagen (B-CTx) were measured by an enzyme-linked immunoassorbent assay. Statistical analyses were performed Prism Version 6.0 Software (Graph Pad Software, Inc., San Diego, CA, USA). The comparison between the groups was performed by ANOVA followed by Sidak's post-test or by Student's t test when appropriate.

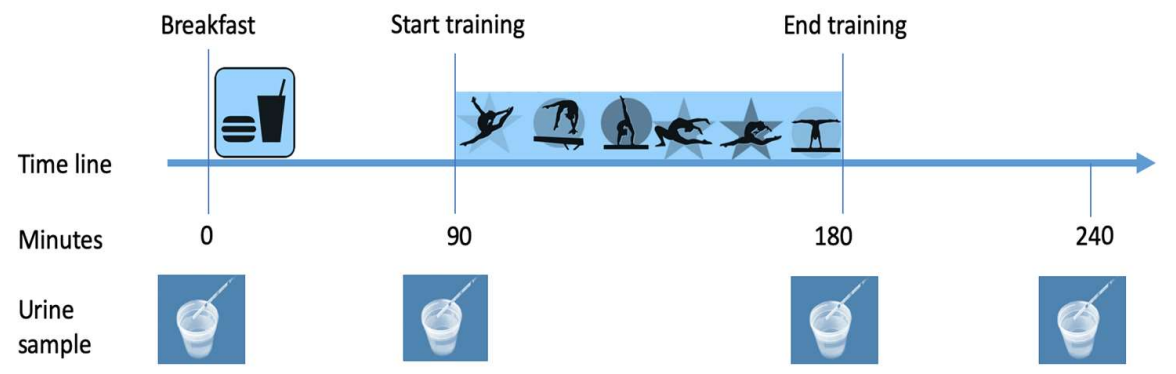

Figure 1. Schematic overview of the experimental protocol. Urine were collected at four different time point. The meal was consumed after the first urine sample was collected. The sport session competition was 90 minutes.

\section{Results and Discussion}

The study analyzed the effects of the pre-exercise meal on bone resorption and the pre-exercise habits. The two groups of gymnasts were similar and not significantly different for age, weight, height, BMI, and body composition or training characteristics. The gymnasts consumed two different pre-exercise isocaloric meal. In particular, the carbohydrates meal provided $88 \%$ of carbohydrates, $9 \%$ of protein and $3 \%$ of fat. The protein meal provided $55 \%$ of carbohydrates, $31 \%$ of protein and $13 \%$ of fat. Our data suggests that the consumption of a pre-exercise meal rich in carbohydrates before the training session significantly attenuates the marker of bone resorption CTX in gymnasts with respect to a meal rich in proteins. In consideration that bone resorption is highly responsive to nutrient ingestion (12-14) it was analyzed frequency, timing of meal consumption and meal type before physical activity in the female child by using a short FFQ to verify if their eating habits were similar to what we propose in the experimental design. We found that about 30 percent of the children do not have regularly a pre-exercise meal and the difference in timing of meal consumption was wide, ranging from 90 to 30 minutes before the training section. We are conscious that the analysis of eating habits pre-exercise was performed in small group of gymnasts and we did not focus the quantity/energy intake of the meal but on frequency and timing of consumption. However, we retain that collect information specifically on the type of meals, frequency and time of consumption in children that perform high impact physical activity could help to verify if is necessary to implement new nutritional intervention.

\section{Conclusion}

The pronounced bone resorption reduction observed in young gymnasts following the consumption of the carbohydrate meal, during the time course, suggests that manipulation of the nutritional composition of the meal influences bone in short term. Interventions for modifying eating habits and meal composition and consumption before physical activity are necessary to improve and preserve child bone health. 
Acknowledgements: We thank the teacher, the parents and the children for their significant contribution.

\section{Reference}

1. Amato A, Sacco A, Macchiarella A, et al. (2017) Influence of nutrition and genetics on performance: a pilot study in a group of gymnasts. Human Movement 18 (3):12-16.

2. Baldassano S, Accardi G, Vasto S (2017) Beta-glucans and cancer: The influence of inflammation and gut peptide. Eur J Med Chem 142:486-492.

3. Baldassano S, Accardi, G, Buscemi, S, et al. (2018) Fibres as functional foods and the effects on gut hormones: the example of $\beta$-glucans in a single arm pilot study. Journal of Functional Foods 47, pp. 264-269

4. Iannaccone, A.; Fusco, A.; Jaime, S.J.; Baldassano, S.; Cooper, J.; Proia, P.; Cortis, C. Stay Home, Stay Active with SuperJump ${ }^{\circledR}$ : A Home-Based Activity to Prevent Sedentary Lifestyle during COVID-19 Outbreak. Sustainability 2020, 12, 10135.

5. Lloyd T, Rollings N, Andon MB, et al. (1992) Determinants of bone density in young women. I. Relationships among pubertal development, total body bone mass, and total body bone density in premenarchal females. J Clin Endocrinol Metab 75 (2):383387.

6. Roemmich JN, Richmond RJ, Rogol AD (2001) Consequences of sport training during puberty. J Endocrinol Invest 24 (9):708715 .

7. Hart E, Meehan WP, Bae DS, et al. (2018) The Young Injured Gymnast: A Literature Review and Discussion. Curr Sports Med Rep 17 (11):366-375.

8. Amato A, Baldassano S ,Cortis C , et al (2018) Physical activity, nutrition and bone health. Human Movement 19, 1-10.

9. Baldassano S, Gasbjerg LS, Kizilkaya HS, et al. (2019) Increased Body Weight and Fat Mass After Subchronic GIP Receptor Antagonist, but Not GLP-2 Receptor Antagonist, Administration in Rats. Front Endocrinol (Lausanne) 10:492.

10. Walsh JS, Henriksen DB (2010) Feeding and bone. Arch Biochem Biophys 503 (1):11-19.

11. Henriksen DB, Alexandersen P, Bjarnason NH, et al. (2003) Role of gastrointestinal hormones in postprandial reduction of bone resorption. J Bone Miner Res 18 (12):2180-2189.

12. Holst J, Hartmann B, Gottschalck IB, et al. (2007) Bone resorption is decreased postprandially by intestinal factors and glucagon-like peptide-2 is a possible candidate. Scand J Gastroenterol 42:814-20. 